nach infolge unserer unseligen Tradition größte Schwierigkeiten. Ich bekam kürzlich im Wege eines Wiederaufnahmeverfahrens den Fall einer Tierärztin auf den Tisch, die sich wegen eines nicht ordnungsgemäß aufgeklärten BSE-Verdachtes auf einem Schlachthof an die Öffentlichkeit gewandt hatte und deswegen fristlos entlassen worden war. Die Entlassung war in zwei Instanzen bestätigt worden. Die grundrechtliche Dimension des Falles ist in keinem der beiden Urteile auch nur angesprochen worden.

Bitte tragen Sie das Ihre dazu bei, daß auch in der Bundesrepublik ein whistleblowerGesetz verabschiedet wird. Es könnte uns wesentlich helfen, Menschen zu schützen, die anderswo in einer kritischen Praxis stehen.

\title{
Reinhard Marx Das Asylrecht - kritisch gesehen
}

Kritisches Rechtsdenken im Asylrecht hat zunächst nach einem rechtlichen Bezugssystem Ausschau zu halten. Die Verfassung hält lediglich den Begriff der politischen Verfolgung bereit. Den Gesetzgeber hat zwar seit Ende der siebziger Jahre eine enorme Regelungswut ergriffen. Die Regelungen betreffen jedoch nahezu ausschließlich verfahrensrechtliche Verschärfungen. Soweit materielle Rechtsfragen gesetzlich geregelt wurden, ging es ausschließlich um negative Abgrenzungskomplexe, wie etwa in Ansehung exilpolitischer Aktivitäten oder der materiellrechtlichen Bedeutung eines Voraufenthaltes in einem Drittstaat während der Flucht. Einen in sich schlüssigen und juristisch anwendungsfähigen Begriff der politischen Verfolgung hat der Gesetzgeber hingegen bislang nicht definiert und wird hiervon wohl auch für die absehbare Zukunft Abstand nehmen.

Das hierdurch entstandene Vakuum hat die Rechtsprechung des Bundesverwaltungsgerichtes ausgefüllt. Bis Ende der siebziger Jahre stellte das Gericht der Verwaltungspraxis geschmeidige Generalklauseln zur Verfügung, mit deren Hilfe mühelos innenund außenpolitischen Interessen Rechnung getragen werden konnte. Seit etwa seit Mitte der siebziger Jahre die Zahl der Flüchtlinge aus der Dritten Welt die der Ostblockflüchtlinge zu übersteigen begann, führte das Bundesverwaltungsgericht jedoch materielle Kriterien in das Asylrecht ein, um hierdurch den personellen Anwendungsbereich des Asylrechts einschneidend zu verengen. Inzwischen bezieht das Bundesverwaltungsgericht sogar mit drohendem Unterton gegen die menschenrechtsfreundliche Rechtsprechung des Europäischen Gerichtshofes für Menschenrechte Position.

Erst seit 1980 hat das Bundesverfassungsgericht seine Rechtsprechung zu materiellen Fragen des Asylrechts entwickelt. Die verfassungsgerichtliche Rechtsprechung zum materiellen Asylrecht ist zwar von einer gemäßigt liberalen Grundhaltung geprägt. Offen bleibt aber auch in der verfassungsgerichtlichen Rechtsprechung, welches Bezugssystem Basis des Asylrechts ist. So hat das Gericht sich zwar wiederholt auf die völkerrechtliche Regelungstradition des Asylrechts bezogen, errichtete dennoch zugleich einen schroffen Dualismus zwischen Verfassungsrecht und Völkerrecht. Vorherrschend ist deshalb bis heute eine scharfe Trennung zwischen dem verfassungsrechtlichen Asylrecht und dem völkerrechtlichen Flüchtlingsrecht. Seit I 994 ist das Bundesverwaltungsgericht sogar dazu übergegangen, anerkannte völkerrechtliche Grundsätze mit einem spezifisch deutschen Bedeutungsgehalt aufzuladen. 
Kritisches Rechtsdenken im Asylrecht kann sich angesichts dessen nicht auf ein anerkanntes Normensystem berufen, anhand dessen die Rechtsprechung auf Methodenklarheit und Plausibilität überprüft werden könnte. Vielmehr entzieht sich insbesondere die Rechtsprechung des Bundesverwaltungsgerichtes durch eine dezisionistisch angelegte Entscheidungspraxis einer Kontrolle. Bei der Behandlung des Bürgerkrieges treibt das Bundesverwaltungsgericht diese Entwicklung auf die Spitze. Einerseits wird diese Rechtsprechung durch den Ausfall methodischer Verläßlichkeit geprägt, so daß individuelle Verfolgungstatbestände mühelos in allgemeine und damit unerhebliche Bürgerkriegsgefahren eingeebnet werden können. Andererseits entwikkelt das Gericht materielle Abgrenzungskriterien, die weder verfassungsrechtlich noch völkerrechtlich zureichend verortet werden und öffnet damit dezisionistischer Willkür Tür und Tor.

Hinzu kommt, daß in der gerichtlichen Praxis der Richter zunehmend durch den Bürokraten abgelöst wird. Nach Maßgabe der revisionsgerichtlichen und der jeweiligen obergerichtlichen Rechtsprechung wird eine Dogmatik entwickelt, die eher der Legitimierung der eigenen Lustlosigkeit am Asylrecht dient, für die besonderen Umstände des Einzelfalles und deren sachgerechte Verarbeitung jedoch kaum noch Sensibilität verspüren läßt. Wenn auch einzuräumen ist, daß der Mißßbrauch des Asylrechts signifikant ist und die häufig gleichbleibenden schematischen Asylbegründungen erhebliche Frustrationsprozesse in der Praxis auslösen, bleibt als menschenrechtliche Forderung an den Rechtsanwender, daß er für den Einzelfall offenbleibt und diesen nicht vorschnell in abstrakte Ablehnungsmuster zwängt. In einem vom allgemeinen Mißtrauen gegen Asylsuchende und Flüchtlinge geprägten gesellschaftlichen Klima ist derart offenes Rechtsdenken jedoch eher der seltene Ausnahmefall.

Da Flüchtlinge darüber hinaus in der politischen Darstellung und medialen Verarbeitung regelmäßig nur noch als illegal Einreisende wahrgenommen werden, prägt der Kampf gegen die illegale Einwanderung das allgemeine Bewußtsein. $\mathrm{Da}$ es legitime Fluchtgründe gibt, wird kaum noch zur Kenntnis genommen. So verstärkt sich das institutionalisierte Mißtrauen gegen Asylsuchende und läßt deren Anerkennungschancen gegen Null sinken. Kritisches Rechtsdenken muß auch diesen von mächtigen politischen und gesellschaftlichen Gruppen gesteuerten Prozeß der Ausgrenzung der Asylsuchenden und Flüchtlinge in den Blick nehmen und darauf insistieren, daß nicht der durch vielfältige Methoden der Kriminalisierung und Stigmatisierung in das Zentrum der Diskussion gerückte Reiseweg, sondern die Verfolgungserlebnisse der Flüchtlinge die Debatte über das Asylrecht beherrschen.

Zentral ist angesichts dieses deprimierenden Befundes die Forderung nach Aufhebung des schroffen Dualismus zwischen dem verfassungsrechtlichen Asylrecht und dem allgemeinen Menschenrechtsschutz. Das politische Asyl ist Teil des allgemeinen Menschenrechtsschutzes. Darauf verweist bereits Artikel I 4 der Allgemeinen Erklärung der Menschenrechte, demzufolge jedermann das Recht hat, in anderen Staaten vor Verfolgung Asyl zu suchen und zu finden. Die Besonderheit des Asylrechts besteht allerdings darin, daß es anders als die allgemeinen Menschenrechte keinen eindeutigen Widerpart hat. Prägt den Menschenrechtsschutz die Beziehung zwischen dem Einzelnen und dem Staat seiner Staatsangehörigkeit, so ist im Blick auf das Asylrecht festzustellen, daß die Staaten es von Anfang an versäumt haben, ein zwischenstaatliches System zu entwickeln, im Rahmen dessen das Asylrecht im Geiste der internationalen Solidarität praktisch Gestalt gewinnen konnte. So blieb von Anfang an die Frage des für die Prüfung des Asylgesuchs und der Asylrechtsgewährung zuständigen Staates offen. 
Das Völkerrecht hat statt dessen nur die individuelle Staatenverantwortlichkeit geregelt: Kein Staat darf einen Flüchtling zwangsweise in einen Staat verbringen, in dem Leib und Leben aus politischen Gründen in Gefahr sind. Welcher Staat für den Schutz des einzelnen Flüchtlings zuständig ist, hat das Völkerrecht mithin nicht geregelt. Fraglich ist allerdings, ob man so weitgehen kann wie das Bundesverfassungsgericht im Urteil zur Drittstaatenregelung, in dem es den Begriff des Viertstaates geprägt hat. Denn kein Staat darf »in irgendeiner Weise« dazu beitragen, daß ein Flüchtling der Gefahr der Abschiebung in seinen Herkunftsstaat ausgesetzt wird. Um dieser Verpflichtung gerecht werden zu können, sind der zurückweisende und der für zuständig angesehene Drittstaat gemeinsam verpflichtet, Schutzvorkehrungen gegen die Gefahr der Weiterschiebung in den Herkunftsstaat zu treffen. Daraus ergibt sich mit zwingender Konsequenz, daß die Reise nicht über den Drittstaat hinausgehen darf. Denn andernfalls kann der zurückweisende Staat keine effektiven Schutzvorkehrungen gegen die Abschiebung in den Herkunftsstaat ergreifen.

Bereits dieses Beispiel belegt, daß das Völkerrecht durchaus sachgerechte Antworten auf viele Problemgestaltungen geben kann. Die augenblickliche materiellrechtliche Verengung des Asylrechts in der Bundesrepublik kann allerdings einen dramatischen Einschnitt in den bisher entwickelten Rechtsstandard des Völkerrechts einleiten. Die Bundesrepublik gehört zu den global playern in den internationalen Staatenbeziehungen. Es besteht deshalb dic Gefahr, daß die durch die Rechtsprechung geprägte Staatenpraxis der Bundesrepublik das Völkerrecht einschneidend verändert. Dies ist insbesondere im Asyl- und Flüchtlingsrecht nicht von der Hand zu weisen, das anders als der allgemeine Menschenrechtsschutz nicht durch internationale, unabhängige Überwachungsorgane ausgelegt wird. Hier werden völkerrechtliche Vertragsnormen weitgehend durch die Staatenpraxis und damit auch durch innerstaatliche Rechtsprechung konkretisiert. Aber auch wenn international berufene Hüter, wie der Straßburger Gerichtshof, die Menschenrechte überwachen, wird diesen inzwischen unverhohlen aus Berlin gedroht, sollten im Ausländerrecht die menschenrechtsfreundlichen Grundsätze weiterhin angewendet werden.

Kritik der herrschenden Rechtspraxis hat im Asylrecht deshalb ihren Ausgang vom menschenrechtlichen Kernbestand des Völkerrechts zu nehmen. Dies erfordert auch für die kritische Rechtstheorie ein Umdenken, da diese regelmäßig Bedeutung und Schutzumfang der Grundrechte vorrangig aus dem Sinnzusammenhang der innerstaatlichen Verfassungsordnung heraus entwickelt, ohne die Verankerung der innerstaatlichen Ordnung im Völkerrecht zu berücksichtigen. Beim Asylrecht, einem die zwischenstaatlichen Beziehungen ins Spiel bringenden Grundrecht, funktioniert diese Methode nicht. Nicht zuletzt die starre Fixierung auf den ausschließlich aus dem innerstaatlichen Kontext heraus definierten alten Artikel 16 Absatz 2 Satz 2 des Grundgesetzes hat die Gegenbewegung gegen die Verfassungsänderung als bloßen, kaum konzeptionell-strategisch ansetzenden Protest erscheinen lassen. Eine wirksame Gegenstrategie gegen innerstaatliche Grundrechtsverkürzungen oder -aufhebungen muß daher völkerrechtlich ansetzen und den Sinngehalt der universellen Menschenrechte für die Schutzverpflichtung gegenüber Flüchtlingen und Asylsuchenden fruchtbar machen. 